\title{
Profile of abemaciclib and its potential in the treatment of breast cancer
}

This article was published in the following Dove Press journal:

OncoTargets and Therapy

\section{James M Martin' \\ Lori J Goldstein ${ }^{2}$}

'Department of Medicine, Section of Hematology/Oncology, Temple University Hospital, Philadelphia, PA, USA; ${ }^{2}$ Department of Medical Oncology, Fox Chase Cancer Center, Philadelphia, PA, USA
Correspondence: James M Martin 333 Cottman Avenue, Philadelphia, PA I 9111 , USA

Email james.martin2@tuhs.temple.edu

\begin{abstract}
Hormone-receptor-positive breast cancer is the most common subtype of breast cancer among patients with both early-stage and metastatic disease. Recent advances in the understanding of its pathophysiology have led to the discovery and utilization of targeted inhibitors to cyclin-dependent kinases 4 and 6 (CDK4/6). There are currently three available CDK4/6 inhibitors available for use in USA: palbociclib, ribociclib, and abemaciclib. Their oral administration and tolerable toxicities make this class of agents appealing to both patients and health care providers. Abemaciclib, the most recently approved CDK4/6 inhibitor, has unique pharmacologic properties and potential toxicities. This review highlights the current understanding of abemaciclib and discusses its current and future roles in the treatment of breast cancer.
\end{abstract}

Keywords: abemaciclib, cyclin D1, breast cancer, CDK4/6

\section{Background}

Breast cancer continues to be the most common malignancy among women and remains a significant cause of mortality. ${ }^{1,2}$ Hormone-receptor-positive $(\mathrm{HR}+)$ breast cancer is the most common subtype of both early-stage and advanced/metastatic disease, representing $\sim 80 \%$ of all breast cancers. ${ }^{3}$ Until recently, the frontline treatment of $\mathrm{HR}+$ advanced/metastatic breast cancer (AMBC) consisted of sequential courses of estrogen blockade. ${ }^{4,5}$ While most patients initially benefit from antiestrogen therapy, the need for cytotoxic chemotherapy is often inevitable. The response to chemotherapy agents is generally poor, ${ }^{6-9}$ indicating the need for additional therapeutic strategies to overcome resistance to endocrine therapy.

Attempts to extend and enhance estrogen therapy have included targeting the phosphatidylinositol 3-kinase-Akt-mammalian target of rapamycin pathway, as demonstrated in the phase III BOLERO-2 study, which showed a progression-free survival (PFS) benefit with the addition of everolimus to exemestane, a steroidal aromatase inhibitor (AI), among women whose disease had progressed on a non-steroidal AI (PFS 6.9 vs 2.8 months; HR, 0.43; 95\% CI, 0.35-0.54; $P<0.001) .{ }^{10}$ Everolimus has also been approved for combination with fulvestrant, an estrogen receptor antagonist. ${ }^{11}$ Additionally, exemestane has been combined with entinostat, a histone deacetylase inhibitor, with encouraging results. ${ }^{12}$

More recently, the promising target among women with AMBC is the inhibition of cell cycle progression by targeting cyclin-dependent kinases 4 and 6 (CDK4/6). In HR+ breast cancer, estrogen stimulates production of the protein cyclin D1, which forms an active complex with CDK4/6. This cyclin-CDK complex then phosphorylates the retinoblastoma $(\mathrm{Rb})$ tumor suppressor protein, which in turn allows for progression through the G1/S transition of the cycle and subsequent cellular proliferation ${ }^{13-15}$. 
Cyclin D1 and CDK4/6 overexpression has been detected in a large portion of breast cancer cases, which can lead to dysregulation of the cell cycle and uncontrolled cellular proliferation. ${ }^{16,17}$ While some studies have reported the overexpression of cyclin D1 as a poor prognostic marker, others have correlated amplification of CCND1, the gene encoding for cyclin D1, as the marker for poor prognosis. ${ }^{15,17,18}$ In preclinical studies, inhibition of CDK4/6 prevents interaction with cyclin D1 and subsequent Rb phosphorylation, leading to cell cycle arrest and restriction of cellular proliferation. ${ }^{19,20}$

There are currently three oral CDK4/6 inhibitors approved by the US Food and Drug Administration (FDA) for women with HR+ AMBC: palbociclib (Ibrance; Pfizer, New York, NY), ribociclib (Kisqali; Novartis, Basel, Switzerland), and abemaciclib (Verzenio; Lilly, Indianapolis, IN). ${ }^{21}$

\section{Palbociclib and ribociclib}

Palbociclib was the first approved oral CDK4/6 inhibitor based on the results of the phase III PALOMA-2 trial, which demonstrated a significant PFS benefit with palbociclib/ letrozole $(\mathrm{N}=444)$ vs letrozole alone $(\mathrm{N}=222)$ among women with previously untreated HR+ AMBC (PFS 22.1 vs 14.5 months; HR $0.58 ; 95 \%$ CI, $0.46-0.72 ; P<0.001) .{ }^{22}$ Its approval was then expanded for use in conjunction with fulvestrant, based on results of the phase III PALOMA-3 trial in which patients were randomized to receive palbociclib/fulvestrant $(\mathrm{N}=347)$ or fulvestrant alone $(\mathrm{N}=174)$ following disease progression on nonsteroidal AI (PFS 9.5 vs 4.6 months; HR 0.46 ; 95\% CI $0.36-0.59 ; P<0.0001) .{ }^{23}$ The most commonly reported grade $3 / 4$ toxicities reported in these studies were neutropenia, leukopenia, and anemia. Neutropenic fever was seen in $1.8 \%$ of patients. PALLAS (NCT02513394) and PATINA (NCT02947685) are ongoing trials examining palbociclib's potential roles in the adjuvant setting or in women with advanced human epidermal growth factor receptor 2 positive (HER2+) breast cancer. ${ }^{24}$

Ribociclib was the second oral CDK4/6 inhibitor approved for frontline AMBC treatment in conjunction with letrozole, based on the phase III MONALEESA-2 study, which similarly demonstrated a PFS benefit in the combination $\operatorname{arm}(\mathrm{N}=334)$ compared with letrozole alone $(\mathrm{N}=334)$ in patients with previously untreated HR+ AMBC (PFS not reached vs 14.7 months; HR 0.59; 95\% CI, 0.41-0.85; $P=0.002) .{ }^{25}$ The most common grade $3 / 4$ toxicities were neutropenia, leukopenia, hypertension, and elevated alanine aminotransferase level. MONALEESA-3 (NCT02422615) explores ribociclib in conjunction with fulvestrant in women with AMBC HR+ breast cancer, previously treated with one prior line of endocrine therapy. MONALEESA-7 (NCT02278120) is an active trial exploring the use of ribociclib in premenopausal women in conjunction with tamoxifen, a selective estrogen receptor modulator. ${ }^{24}$

\section{Abemaciclib}

Abemaciclib has been approved for frontline and later treatment of $\mathrm{HR}+\mathrm{AMBC}$, both in conjunction with antiestrogen therapy and as monotherapy (Tables 1 and 2). In comparison with palbociclib and ribociclib, abemaciclib has a higher potency and greater specificity for CDK4 based on preclinical pharmacokinetic models, although there are no clinical data demonstrating that this impacts clinical outcome. ${ }^{26,27}$ Also in contrast to palbociclib and ribociclib, which are both taken on days 1-21 of a 28-day cycle due to myelosuppression, abemaciclib is given on a continuous dosing schedule. ${ }^{28-30}$ In vitro studies have also demonstrated that abemaciclib is a competitive inhibitor of ATP-binding cassette transporters, particularly ABCB1 and ABCG2, which play a role in the development of chemotherapeutic resistance. ${ }^{31}$ Further studies are required to determine whether this property has any clinical impact.

Table I Comparison of approved use and reported efficacy

\begin{tabular}{|c|c|c|c|}
\hline Drug characteristic & Abemaciclib & Ribociclib & Palbociclib \\
\hline Initial standard dosing & $\begin{array}{l}200 \mathrm{mg} \text { twice daily as monotherapy; } 150 \mathrm{mg} \\
\text { twice daily as combination therapy }\end{array}$ & $\begin{array}{l}600 \mathrm{mg} \text { daily (days }|-2| \text { of } \\
\text { a } 28-\text { day cycle) }\end{array}$ & $\begin{array}{l}\text { I } 25 \mathrm{mg} \text { daily (days }|-2| \text { of } \\
\text { a } 28 \text {-day cycle) }\end{array}$ \\
\hline FDA approval first line (AMBC) & Yes, in combination with $\mathrm{Al}$ & Yes, in combination with $\mathrm{Al}$ & Yes, in combination with $\mathrm{Al}$ \\
\hline FDA approval second line (AMBC) & Yes, in combination with fulvestrant & No & $\begin{array}{l}\text { Yes, in combination with } \\
\text { fulvestrant }\end{array}$ \\
\hline FDA approval as monotherapy (AMBC) & $\begin{array}{l}\text { Yes, after progression on endocrine } \\
\text { therapy and any chemotherapy }\end{array}$ & No & No \\
\hline $\begin{array}{l}\text { Median PFS (months) in combination } \\
\text { with } \mathrm{Al} \text { vs } \mathrm{Al} \text { alone }\end{array}$ & NR vs 14.7 & NR vs 14.7 & 24.8 vs 14.5 \\
\hline $\begin{array}{l}\text { Median PFS (months) in combination } \\
\text { with fulvestrant vs fulvestrant alone }\end{array}$ & 16.4 vs 9.3 & N/A & 9.5 vs 4.6 \\
\hline
\end{tabular}

Abbreviations: FDA, Food and Drug Administration; AMBC, advanced or metastatic breast cancer; Al, aromatase inhibitor; PFS, progression-free survival; NR, not reached; $\mathrm{N} / \mathrm{A}$, not available. 
Table 2 Most commonly reported grade 3 or 4 toxicities

\begin{tabular}{lll}
\hline Abemaciclib & Ribociclib & Palbociclib \\
\hline Neutropenia & Neutropenia & Neutropenia \\
Diarrhea & Leukopenia & Leukopenia \\
Leukopenia & Abnormal LFTs & Infections \\
Anemia & Vomiting & Anemia \\
\hline
\end{tabular}

Abbreviation: LFTs, liver function tests.

Here, we will review the role of abemaciclib as both monotherapy and combination therapy for $\mathrm{HR}+\mathrm{AMBC}$, as well as the ongoing investigations of its potential utilization for central nervous system metastases HER2+ disease, triplenegative breast cancer (TNBC), and early breast cancer.

\section{Abemaciclib in advanced $\mathbf{H R}+$ breast cancer Monotherapy}

The safety and efficacy of abemaciclib as a single agent were established in a phase I clinical trial that included 225 patients with advanced solid tumors. ${ }^{30}$ This included a dose escalation phase consisting of 33 patients during which both daily and twice-daily (Q12H) dosing schedules were evaluated. Abemaciclib was given continuously on days 1 through 28 of a 28 -day cycle. The maximum tolerated dose (MTD) of the daily dosing schedule was not reached, whereas the MTD for twice-daily dosing was established at $200 \mathrm{mg}$ Q12H. The terminal elimination half-life ranged between 17.4 and 38.1 hours. Both $150 \mathrm{mg}$ Q12H and $200 \mathrm{mg}$ Q12H were investigated in the subsequent tumor-specific cohorts. In contrast to palbociclib and ribociclib, the dose-limiting toxicity was fatigue rather than neutropenia. ${ }^{28,29}$

Tumor-specific cohorts were investigated in breast cancer ( $\mathrm{N}=66)$, non-small-cell lung cancer $(\mathrm{N}=68)$, glioblastoma $(\mathrm{N}=17)$, melanoma $(\mathrm{N}=26)$, and colorectal cancer $(\mathrm{N}=15){ }^{30}$ Measurable disease was required, and tumor responses were evaluated every two cycles by imaging, using Response Evaluation Criteria In Solid Tumors (RECIST), version 1.1. A total of 47 patients with $A M B C$ received single-agent abemaciclib; $\mathrm{HR}+(\mathrm{N}=36)$, hormone-receptor-negative (HR-; $\mathrm{N}=9$ ), and HR-unknown $(\mathrm{N}=2)$ diseases were included in this cohort. An additional 19 patients with HR+ breast cancer received abemaciclib in combination with fulvestrant. Both breast cancer cohorts were heavily pretreated, with $52 \%$ and $63 \%$ of patients having received greater than or equal to four prior systemic therapy regimens in the single-agent and combination cohorts, respectively. No prior exposure to CDK4/6 inhibitors was allowed.

Among the breast cancer patients treated with singleagent abemaciclib, the partial response (PR) rate was $23 \%$, and $47 \%$ of patients achieved stable disease (SD). ${ }^{30}$ There were no complete responses (CRs) observed. The clinical benefit rate $(\mathrm{CR}+\mathrm{PR}+\mathrm{SD} \geq 24$ weeks) was $49 \%$. Among the patients with $\mathrm{HR}+$ breast cancer, the single-agent clinical benefit rate was higher at $61.1 \%$ compared with $11.1 \%$ of the patients with HR- disease. Treatment effect was also noted in patients with $\mathrm{HR}+/ \mathrm{HER} 2+$ breast cancer $(\mathrm{N}=11)$. In the additional cohort that combined abemaciclib with fulvestrant for HR+ disease, a clinical benefit rate of $63 \%$ was seen.

Among the other tumor types included within the study, the response rates were less pronounced than those seen among the patients with breast cancer. ${ }^{30}$ The disease control rates $(\mathrm{CR}+\mathrm{PR}+\mathrm{SD})$ among patients with lung cancer, glioblastoma, colorectal cancer, and melanoma were $49 \%, 18 \%$, $13 \%$, and $27 \%$, respectively.

The most common toxicities for all patients who received single-agent abemaciclib within a tumor-specific cohort ( $\mathrm{N}=173$ ) were diarrhea, nausea, and fatigue. ${ }^{30}$ Grade 1-2 diarrhea was reported in $58 \%$ of all patients in tumorspecific cohorts. Five percent of patients reported grade 3 diarrhea; there was no grade 4 diarrhea reported. No patients discontinued therapy due to diarrhea. Grade 3-4 neutropenia occurred in $10 \%$ of patients in tumor-specific cohorts, with only one neutropenic fever reported $(0.6 \%)$.

Among the 19 patients treated with combination abemaciclib and fulvestrant, the most common toxicities were diarrhea, fatigue, and nausea. ${ }^{30}$ Diarrhea was reported in $79 \%$ of patients, with grade 3 diarrhea reported in one patient (5\%). There was no grade 4 diarrhea reported. Grade 3 neutropenia was reported in six patients (32\%), and no grade 4 neutropenia or neutropenic fever was reported.

Pharmacodynamic monitoring was performed in both the dose escalation and tumor-specific cohorts by measurement of phosphorylated RB (pRB) and topoisomerase II alpha (TopoII $\alpha$ ) within epidermal keratinocytes. ${ }^{30}$ Reduced levels of $\mathrm{pRB}$ and TopoII $\alpha$ were seen at both $150 \mathrm{mg}$ and $200 \mathrm{mg}$ Q12H. These effects were observed both predose and postdose, indicating a steady state of target effect from abemaciclib at both dosing schedules.

Abemaciclib's efficacy in breast cancer was further established in MONARCH 1, a phase II single-arm multicenter trial. ${ }^{32}$ Eligible patients had HR+ metastatic breast cancer with measurable disease that had progressed on or after prior endocrine therapy and had prior treatment with one or two chemotherapy regimens in the metastatic setting. Additionally, a taxane must have been included in either the adjuvant or metastatic setting. Patients were excluded if they had prior exposure to a CDK4/6 inhibitor. 
A total of 132 patients were enrolled and treated with abemaciclib $200 \mathrm{mg}$ Q12H. ${ }^{32}$ The PR rate was 19.7\%, and there were no CRs observed. The clinical benefit rate was $42.4 \%$. With an 18-month follow-up period, the median OS was estimated at 22.3 months with a mean PFS of 18 months.

Treatment-related adverse events (AEs) were experienced in all patients. ${ }^{32}$ The most common AE was diarrhea, which was experienced by $119(90.2 \%)$ patients. Grade 1 and grade 2 diarrhea occurred in $41.7 \%$ and $28.8 \%$ of patients, respectively. The median time of onset of diarrhea was 7 days into initiation of abemaciclib therapy. Grade 3 diarrhea occurred less commonly, ie, reported in $19.7 \%$ of patients. No grade 4 diarrhea was reported. Neutropenia occurred in $87.7 \%$ of patients, with $22.3 \%$ and $4.6 \%$ of patients experiencing grade 3 and grade 4 neutropenia, respectively. One patient experienced febrile neutropenia, although this occurred 19 days after discontinuing abemaciclib and 8 days after starting treatment with fluorouracil and vinorelbine.

Elevations in serum creatinine were also reported in $98.5 \%$ of patients. $^{32}$ Only $0.8 \%$ of patients had grade 3 elevation, and there were no grade 4 elevations reported. The elevated creatinine levels remained stable throughout the dosing period and decreased at the short-term follow-up visit that occurred once study treatment was discontinued. Increases in blood urea nitrogen were not reported. Measurements of cystatin $\mathrm{C}$, an alternative measurement of glomerular filtration rate, were not concurrently elevated, indicating that the overall renal function was not significantly impaired. One patient discontinued treatment due to creatinine elevation. The elevation in creatinine is due to abemaciclib's inhibitory effects on renal efflux transporters, which are also responsible for active secretion of creatinine..$^{30}$

Dose reductions due to AEs occurred in $49.2 \%$ of patients. ${ }^{32}$ The most common AEs requiring dose reduction were diarrhea and neutropenia. Dose omissions due to AEs occurred in $57.6 \%$ of patients and were most commonly due to diarrhea and neutropenia. Only $7.6 \%$ of patients discontinued study due to AEs.

Single-agent abemaciclib (200 mg Q12H) was approved by the FDA on September 28, 2017, for women with HR+ AMBC progressing on both endocrine therapy and any chemotherapy, despite high dose reduction/omission rates seen in MONARCH 1 at this dosage level. ${ }^{32}$ In addition, the National Comprehensive Cancer Network (NCCN) has added monotherapy with abemaciclib to its breast cancer guidelines for treatment following progression on endocrine therapy and any chemotherapy. ${ }^{4}$

\section{Combination therapy}

Abemaciclib's efficacy and safety in combination with antiestrogen therapy were established in two large, multicenter clinical trials. MONARCH 2 was a phase III, randomized, double-blind study examining fulvestrant/abemaciclib vs fulvestrant/placebo. ${ }^{33}$ Eligible patients were women with $\mathrm{HR}+\mathrm{AMBC}$ whose disease had progressed while receiving prior endocrine therapy or within 12 months of completing adjuvant endocrine therapy. Patients could not have received more than one line of endocrine therapy, prior chemotherapy for metastatic disease, fulvestrant, everolimus, or previous CDK4/6 inhibition. The study's primary endpoint was PFS.

A total of 669 patients were enrolled, and $25.3 \%$ were deemed to have primary endocrine therapy resistance. ${ }^{33}$ Primary endocrine therapy resistance was defined as disease that relapsed while receiving the first 2 years of adjuvant/ neoadjuvant endocrine therapy or disease that progressed within the first 6 months of endocrine therapy for AMBC. At initiation of the study, the dose of abemaciclib was $200 \mathrm{mg}$ Q12H, but this was eventually reduced to $150 \mathrm{mg}$ Q12H after a review of safety data and dose reduction rates. The CR and PR rates were $3.1 \%$ and $32.1 \%$ for the abemaciclib group, respectively, compared with $0.4 \%$ and $15.7 \%$ in the placebo group. The clinical benefit rate was $72.2 \%$ for abemaciclib and $56.1 \%$ for placebo. Median PFS was found to be 16.4 months for the abemaciclib group and 9.3 months for the placebo group (HR 0.553; 95\% CI, 0.449-0.681; $P<0.001$ ).

Observed toxicities were similar to those seen in MON$\mathrm{ARCH} 1$, with diarrhea, neutropenia, nausea, and fatigue listed as the most common treatment-related AEs. ${ }^{33}$ Diarrhea was by far the most common AE, with $86.4 \%$ of patients in the abemaciclib group reporting diarrhea compared with $24.7 \%$ in the placebo group. As in MONARCH 1, a majority of diarrhea was grades 1 and 2, with $13.4 \%$ of patients experiencing grade 3 diarrhea. No grade 4 diarrhea was reported. The diarrhea within the abemaciclib group was effectively managed with antidiarrheal medications, and $70.1 \%$ of patients who experienced diarrhea did not require treatment modification. Neutropenia occurred in $46 \%$ of patients receiving abemaciclib compared with $4 \%$ receiving placebo. Febrile neutropenia was reported in six patients in the abemaciclib arm (1.3\%), although one of these patients had grade 2 afebrile neutropenia that was incorrectly coded and another patient had febrile neutropenia in the study follow-up period after discontinuation of abemaciclib and receipt of paclitaxel.

Abemaciclib, in combination with fulvestrant, was approved by the FDA on September 28, 2017, for the 
treatment of $\mathrm{HR}+\mathrm{AMBC}$ with progression on antiestrogen therapy. Combination therapy with fulvestrant has also been added to the NCCN guidelines for metastatic HR+ breast cancer following progression on initial endocrine therapy. ${ }^{4}$

MONARCH 3 was a phase III, randomized, double-blind study that randomized previously untreated women with $\mathrm{HR}+$ AMBC to receive a nonsteroidal AI with either abemaciclib or placebo. ${ }^{34}$ Prior endocrine therapy in the neoadjuvant or adjuvant setting was permitted if the patient had a diseasefree interval of $>12$ months from its completion. Patients may not have received any systemic therapy for advanced disease or have any prior exposure to CDK4/6 inhibitors. Again, the study's primary objective was PFS.

A total of 493 patients were enrolled and randomized to receive a nonsteroidal AI (either anastrozole or letrozole, per physician choice) with abemaciclib or placebo. ${ }^{34}$ Abemaciclib was given at a dose of $150 \mathrm{mg}$ Q12H. The study met its primary endpoint with a median PFS not reached in the abemaciclib arm compared with 14.7 months in the placebo arm (HR 0.54; 95\% CI, 0.41-0.72; $P=0.000021$ ). The CR and $\mathrm{PR}$ rates were $1.5 \%$ and $46.6 \%$ in the abemaciclib arm, respectively, compared with $0 \%$ and $34.5 \%$ in the placebo arm. The clinical benefit rate was $78 \%$ for the abemaciclib arm and $71.5 \%$ for the placebo arm.

The toxicity profile for abemaciclib plus nonsteroidal AI was similar to that seen in MONARCH 2 with regard to gastrointestinal toxicity, neutropenia, and creatinine elevation. ${ }^{34}$ There was, however, a higher rate of venous thromboembolism in the abemaciclib arm at $4.9 \%$ compared with $0.6 \%$ in the placebo arm. A total of $19.6 \%$ of abemaciclib patients discontinued treatment due to treatment-related AEs compared with $2.5 \%$ in the placebo arm. The discontinuation rate for abemaciclib due to diarrhea was $2.3 \%$.

On February 26, 2018, the FDA approved abemaciclib in combination with AI for frontline treatment of metastatic HR+ breast cancer. Combination therapy with AI has also been added to the NCCN guidelines as a possible treatment regimen for frontline treatment of metastatic HR+ breast cancer. ${ }^{4}$

\section{Biomarkers of response}

Currently, there are no known biomarkers that will predict which patients will benefit from CDK4/6 inhibition. Preclinical data with palbociclib suggested that high levels of both cyclin D1 and $\mathrm{Rb}$, as well as low levels of p16, a tumor suppressor protein that inhibits CDK4/6, were predictors of response. ${ }^{35}$ Human tumor xenografts have demonstrated that loss of $\mathrm{Rb}$ leads to resistance to CDK4/6 inhibition. ${ }^{19,26,36}$ However, loss of $\mathrm{Rb}$ in $\mathrm{HR}+$ breast cancers is a rare event. ${ }^{16}$ Moreover, in the phase II PALOMA-1 study involving palbociclib, there was no difference in response to treatment for patients with cyclin D1 amplification or p16 loss compared with the overall study population. ${ }^{37}$ For this study, cyclin D1 amplification and p16 loss were determined by central laboratory verification utilizing a CCND1-to-chromosome enumeration probe (CEP) 11 ratio $>1.5$, and p16 loss as a p16-to-CEP9 ratio of $<0.8$. Additional insights into the cell cycle are required to select patients who will benefit from CDK4/6-directed therapy.

\section{Central nervous system metastases}

Pharmacokinetic testing during the original phase I study for abemaciclib demonstrated significant drug present within the cerebrospinal fluid of the study patients, which approximated plasma concentrations. ${ }^{30}$ NCT02308020 is an ongoing twostage, phase II study evaluating abemaciclib $200 \mathrm{mg} \mathrm{Q12H}$ in patients with $\mathrm{HR}+\mathrm{AMBC}$, non-small-cell lung cancer, or melanoma, with at least one measurable brain metastasis. ${ }^{24}$ A stage 1 efficacy analysis of the HR+/HER2- AMBC cohort demonstrated an adequate objective intracranial response to proceed with further enrollment. ${ }^{38}$ The study is projected to enroll 247 patients.

\section{HER2+ disease}

Abemaciclib monotherapy demonstrated efficacy among patients with $\mathrm{HR}+/ \mathrm{HER} 2+$ in the original phase I study. ${ }^{30} \mathrm{Of}$ the 11 patients with HER2+ disease, the clinical benefit rate was $54.5 \%$. Four patients (36\%) achieved a PR. This suggests that abemaciclib is likely active in patients with HER2+ breast cancer, despite the small number of study subjects. In addition, there appears to be synergy between CDK4/6 inhibition and anti-HER2 therapy in vitro. ${ }^{39}$ NCT02675231 (monarcHER) is an ongoing randomized phase II clinical trial for which patients with HR+/HER2+ AMBC are randomized $1: 1: 1$ to receive abemaciclib plus trastuzumab with or without fulvestrant vs standard-of-care chemotherapy plus trastuzumab. ${ }^{24}$ Eligible patients must have received at least two different HER2-directed therapies and a taxane. The trial is expected to enroll 225 patients.

\section{Triple-negative disease}

In the original phase I study, abemaciclib's clinical benefit rate as monotherapy was low at $11.1 \%$ among the nine patients with $\mathrm{HR}$ - breast cancer. ${ }^{30}$ No patients achieved a CR or PR. However, there is a known subset of TNBC that is characterized by the androgen receptor (AR) signaling, which is more likely to express $\mathrm{Rb} .{ }^{40,41}$ AR-expressing TNBC has been shown to be quite sensitive to CDK4/6 
inhibition in xenograft models, ${ }^{42}$ indicating potential benefit from abemaciclib or the other available CDK4/6 inhibitors. NCT03130439 is an ongoing phase II study evaluating the activity of abemaciclib in patients with $\mathrm{Rb}+\mathrm{TNBC}$ who have received prior cytotoxic chemotherapy. ${ }^{24}$ Thirty-seven patients are expected to enroll.

\section{Early breast cancer}

Despite appropriate administration of adjuvant (or neoadjuvant) endocrine therapy, many patients with early HR+ breast cancer will eventually relapse. ${ }^{43} \mathrm{CDK} 4 / 6$ inhibition in the adjuvant or neoadjuvant period provides a potential option for improvement in relapse rates among patients with early disease. The neoMONARCH study (NCT02441946) randomized 223 postmenopausal women with HR+/HER2- early breast cancer to receive neoadjuvant anastrozole, abemaciclib, or both for 2 weeks. Patients then received 14 weeks of both drugs followed by optional surgery. ${ }^{24}$ The primary endpoint of the study was change from baseline to 2 weeks in expression of Ki67, a marker of cell proliferation, which may be predictive of improved disease-free survival. ${ }^{44}$ Preliminary data presented at the 2017 San Antonio Breast Cancer Symposium showed that $57.7 \%$ of patients who received abemaciclib achieved cell cycle arrest (defined as Ki67 <2.7\%) compared with $14.3 \%$ of patients who received anastrozole alone. ${ }^{45}$

The monarchE study (NCT03155997) has been designed to examine abemaciclib in the adjuvant setting. ${ }^{24}$ Women with high-risk, node-positive, early HR+/HER2- breast cancer will be randomized to receive standard adjuvant endocrine therapy with or without abemaciclib. A total of 3,580 patients are expected to enroll.

\section{Conclusion}

CDK4/6 inhibitors are an effective and promising option for the treatment of advanced or metastatic HR+ breast cancer. Their toxicity profile and oral administration are convenient and manageable. Abemaciclib is currently FDA-approved for $\mathrm{HR}+\mathrm{AMBC}$ in combination with endocrine therapy in the first- and second-line setting, and it is the only CDK4/6 inhibitor approved as monotherapy following progression on both endocrine therapy and chemotherapy. Future research directions include determining whether CDK4/6 inhibition should be continued beyond disease progression, potential utility in early breast cancer and central nervous system disease, biomarker selection, and novel combinations with other classes of agents. It is also unknown whether abemaciclib has activity in patients who have been treated with another CDK4/6 inhibitor. Abemaciclib appears to possess unique pharmacologic properties, and additional studies will likely show further utility within the therapeutic arsenal for breast cancer and potentially other types of malignancies.

\section{Disclosure}

LJG holds consultant/advisory roles with PUMA Biotechnologies, Dompe, Genentech, Novartis, and Genomic Health. JMM has no conflicts of interest in this work.

\section{References}

1. U.S. Cancer Statistics Working Group. United States Cancer Statistics: 1999-2014 Incidence and Mortality Web-based Report. Atlanta: U.S. Department of Health and Human Services, Centers for Disease Control and Prevention and National Cancer Institute. 2017. [cited November 28, 2017]. Available from: www.cdc.gov/uscs

2. National Cancer Institute. SEER Cancer Statistics Factsheets: Breast Cancer. [cited November 28, 2017]. Available from: https://seer.cancer. gov/statfacts $/ \mathrm{html} /$ breast.html

3. Kohler BA, Sherman RL, Howlader N, et al. Annual Report to the Nation on the Status of Cancer, 1975-2011, Featuring Incidence of Breast Cancer Subtypes by Race/Ethnicity, Poverty, and State. J Natl Cancer Inst. 2015;107(6):djv048.

4. National Comprehensive Cancer Network. Breast Cancer. Available from: https://www.nccn.org/professionals/physician_gls/pdf/breast.pdf. Accessed March 22, 2018.

5. Partridge AH, Rumble RB, Carey LA, et al. Chemotherapy and targeted therapy for women with human epidermal growth factor receptor 2-negative (or unknown) advanced breast cancer: American Society of Clinical Oncology Clinical Practice Guideline. J Clin Oncol. 2014;32(29):3307-3329.

6. Sledge GW, Neuberg D, Bernardo P, et al. Phase III trial of doxorubicin, paclitaxel, and the combination of doxorubicin and paclitaxel as front-line chemotherapy for metastatic breast cancer: an intergroup trial (E1193). J Clin Oncol. 2003;21(4):588-592.

7. Cortes J, O'Shaughnessy J, Loesch D, et al. Eribulin monotherapy versus treatment of physician's choice in patients with metastatic breast cancer (EMBRACE): a phase 3 open-label randomised study. Lancet. 2011;377(9769):914-923.

8. Perez EA, Lerzo G, Pivot X, et al. Efficacy and safety of ixabepilone (BMS-247550) in a phase II study of patients with advanced breast cancer resistant to an anthracycline, a taxane, and capecitabine. J Clin Oncol. 2007;25(23):3407-3414.

9. Kaufman PA, Awada A, Twelves C, et al. Phase III open-label randomized study of eribulin mesylate versus capecitabine in patients with locally advanced or metastatic breast cancer previously treated with an anthracycline and a taxane. J Clin Oncol. 2015;33(6):594-601.

10. Baselga J, Campone M, Piccart M, et al. Everolimus in postmenopausal hormone-receptor-positive advanced breast cancer. N Engl J Med. 2012; 366(6):520-529.

11. Kornblum NS, Zhao F, Manola J, et al. Randomized phase II trial of fulbestrant plus everolimus or placebo in post-menopausal women with hormone-receptor positive HER2-negative metastatic breast cancer resistant to aromatase inhibitor therapy. J Clin Oncol. 2012;30(22): 2718-2724.

12. Yardley DA, Ismail-Khan RR, Melichar B, et al. Randomized phase II, double-blind, placebo-controlled study of exemestane with or without entinostat in postmenopausal women with locally recurrent or metastatic estrogen receptor-positive breast cancer progressing on treatment with a nonsteroidal aromatase inhibitor. J Clin Oncol. 2013; 31(17):2128-2135.

13. Vanarsdale T, Boshoff C, Arndt KT, Abraham RT. Molecular pathways: targeting the cyclin D-CDK4/6 axis for cancer treatment. Clin Cancer Res. 2015;21(13):2905-2910.

14. Barnes DM, Gillett CE. Cyclin D1 in breast cancer. Breast Cancer Res Treat. 1998;52(1-3):1-15. 
15. Lundgren K, Brown M, Pineda S, et al. Effects of cyclin D1 gene amplification and protein expression on time to recurrence in postmenopausal breast cancer patients treated with anastrozole or tamoxifen: a TransATAC study. Breast Cancer Res. 2012;14(2):R57.

16. Cancer Genome Atlas Network. Comprehensive molecular portraits of human breast tumours. Nature. 2012;490(7418):61-70.

17. Stendahl M, Kronblad A, Rydén L, et al. Cyclin D1 overexpression is a negative predictive factor for tamoxifen response in postmenopausal breast cancer patients. Br J Cancer. 2004;90(10):1942-1948.

18. Naidu R, Wahab NA, Yadav MM, Kutty MK. Expression and amplification of cyclin D1 in primary breast carcinomas: relationship with histopathological types and clinico-pathological parameters. Oncol Rep. 2002;9(2):409-416.

19. Dean JL, Thangavel C, Mcclendon AK, Reed CA, Knudsen ES. Therapeutic CDK4/6 inhibition in breast cancer: key mechanisms of response and failure. Oncogene. 2010;29(28):4018-4032.

20. Finn RS, Aleshin A, Slamon DJ. Targeting the cyclin-dependent kinases (CDK) 4/6 in estrogen receptor-positive breast cancers. Breast Cancer Res. 2016;18(1):17.

21. Xu H, Yu S, Liu Q, et al. Recent advances of highly selective CDK4/6 inhibitors in breast cancer. J Hematol Oncol. 2017;10(1):97.

22. Finn RS, Martin M, Rugo HS, et al. Palbociclib and letrozole in advanced breast cancer. N Engl J Med. 2016;375(20):1925-1936.

23. Cristofanilli M, Turner NC, Bondarenko I, et al. Fulvestrant plus palbociclib versus fulvestrant plus placebo for treatment of hormonereceptor-positive, HER2-negative metastatic breast cancer that progressed on previous endocrine therapy (PALOMA-3): final analysis of the multicentre, double-blind, phase 3 randomised controlled trial. Lancet Oncol. 2016;17(4):425-439.

24. Available from: https://clinicaltrials.gov. Accessed November 27, 2017.

25. Hortobagyi GN, Stemmer SM, Burris HA, et al. Ribociclib as firstline therapy for HR-positive, advanced breast cancer. $N$ Engl J Med. 2016;375(18):1738-1748.

26. Gelbert LM, Cai S, Lin X, et al. Preclinical characterization of the CDK4/6 inhibitor LY2835219: in-vivo cell cycle-dependent/independent anti-tumor activities alone/in combination with gemcitabine. Invest New Drugs. 2014;32(5):825-837.

27. Tate SC, Cai S, Ajamie RT, et al. Semi-mechanistic pharmacokinetic/ pharmacodynamic modeling of the antitumor activity of LY2835219, a new cyclin-dependent kinase 4/6 inhibitor, in mice bearing human tumor xenografts. Clin Cancer Res. 2014;20(14):3763-3774.

28. Flaherty KT, Lorusso PM, Demichele A, et al. Phase I, dose-escalation trial of the oral cyclin-dependent kinase 4/6 inhibitor PD 0332991, administered using a 21 -day schedule in patients with advanced cancer. Clin Cancer Res. 2012;18(2):568-576.

29. Infante JR, Cassier PA, Gerecitano JF, et al. A phase I study of the cyclin-dependent kinase 4/6 inhibitor ribociclib (LEE011) in patients with advanced solid tumors and lymphomas. Clin Cancer Res. 2016; 22(23):5696-5705

30. Patnaik A, Rosen LS, Tolaney SM, et al. Efficacy and safety of abemaciclib, an inhibitor of CDK4 and CDK6, for patients with breast cancer, non-small cell lung cancer, and other solid tumors. Cancer Discov. 2016;6(7):740-753.

OncoTargets and Therapy

\section{Publish your work in this journal}

OncoTargets and Therapy is an international, peer-reviewed, open access journal focusing on the pathological basis of all cancers, potential targets for therapy and treatment protocols employed to improve the management of cancer patients. The journal also focuses on the impact of management programs and new therapeutic agents and protocols on
31. Wu T, Chen Z, To KKW, et al. Effect of abemaciclib (LY2835219) on enhancement of chemotherapeutic agents in ABCB1 and ABCG2 overexpressing cells in vitro and in vivo. Biochem Pharmacol. 2017; 124:29-42.

32. Dickler MN, Tolaney SM, Rugo HS, et al. MONARCH 1, A phase II study of abemaciclib, a CDK4 and CDK6 inhibitor, as a single agent, in patients with refractory $\mathrm{HR}^{+} / \mathrm{HER} 2^{-}$metastatic breast cancer. Clin Cancer Res. 2017;23(17):5218-5224.

33. Sledge GW, Toi M, Neven P, et al. MONARCH 2: abemaciclib in combination with fulvestrant in women with HR+/HER2- advanced breast cancer who had progressed while receiving endocrine therapy. J Clin Oncol. 2017;35(25):2875-2884.

34. Goetz MP, Toi M, Campone M, et al. MONARCH 3: abemaciclib as initial therapy for advanced breast cancer. J Clin Oncol. 2017;35(32): 3638-3646.

35. Finn RS, Dering J, Conklin D, et al. PD 0332991, a selective cyclin D kinase 4/6 inhibitor, preferentially inhibits proliferation of luminal estrogen receptor-positive human breast cancer cell lines in vitro. Breast Cancer Res. 2009;11(5):R77.

36. Fry DW, Harvey PJ, Keller PR, et al. Specific inhibition of cyclin-dependent kinase 4/6 by PD 0332991 and associated antitumor activity in human tumor xenografts. Mol Cancer Ther. 2004;3(11):1427-1438.

37. Finn RS, Crown JP, Lang I, et al. The cyclin-dependent kinase $4 / 6$ inhibitor palbociclib in combination with letrozole versus letrozole alone as first-line treatment of oestrogen receptor-positive, HER2-negative, advanced breast cancer (PALOMA-1/TRIO-18): a randomised phase 2 study. Lancet Oncol. 2015;16(1):25-35.

38. Tolaney SM, Lin NU, Thornton D, et al. Abemaciclib for the treatment of brain metastases (BM) secondary to hormone receptor positive $(\mathrm{HR}+)$, HER2 negative breast cancer. J Clin Oncol. 2017 ; 35(15_suppl):1019-1019.

39. Goel S, Wang Q, Watt AC, et al. Overcoming therapeutic resistance in HER2-positive breast cancers with CDK4/6 inhibitors. Cancer Cell. 2016;29(3):255-269.

40. Lehmann BD, Bauer JA, Chen X, et al. Identification of human triplenegative breast cancer subtypes and preclinical models for selection of targeted therapies. J Clin Invest. 2011;121(7):2750-2767.

41. Patel JM, Torous V, Hacker MR, et al. Retinoblastoma ( $\mathrm{Rb}$ ) protein expression in triple-negative breast cancer. J Clin Oncol. 2017 35(15_suppl):1097-1097.

42. Asghar US, Barr AR, Cutts R, et al. Single-cell dynamics determines response to $\mathrm{CDK} 4 / 6$ inhibition in triple-negative breast cancer. Clin Cancer Res. 2017;23(18):5561-5572.

43. Pan H, Gray R, Braybrooke J, et al. 20-Year risks of breast-cancer recurrence after stopping endocrine therapy at 5 years. $N$ Engl J Med. 2017; 377(19):1836-1846.

44. Yoshioka T, Hosoda M, Yamamoto M, et al. Prognostic significance of pathologic complete response and Ki67 expression after neoadjuvant chemotherapy in breast cancer. Breast Cancer. 2015;22(2):185-191.

45. Martin M, Hurvitz SA, Chan D, et al. Final results of NeoMONARCH: A phase 2 neoadjuvant study of abemaciclib in postmenopausal women with hormone receptor positive (HR+), HER2 negative breast cancer (BC). Presented at: the 2017 San Antonio Breast Cancer Symposium; December 5-9, 2017. Abstract PD5-01.

patient perspectives such as quality of life, adherence and satisfaction The manuscript management system is completely online and includes a very quick and fair peer-review system, which is all easy to use. Visit http://www.dovepress.com/testimonials.php to read real quotes from published authors. 